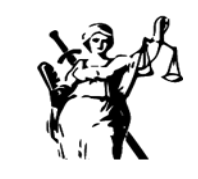

JUSTICIA

ISSN impreso 0124-7441
ISSN digital 2590-4566

\title{
Análisis jurídico del régimen de pensiòn en trabajadores agrícolas en relación con el trabajo decente*
}

\author{
Legal analysis of the pension scheme in agricultural workers in \\ relation to decent work
}

\author{
Hugo Armando Ramirez Acevedo \\ Universidad de Boyacá, Colombia \\ hugramirez@uniboyaca.edu.co
}

\author{
Paula Stefany Pérez Romero \\ Universidad de Boyacá, Colombia \\ paula.perez.z.pr@gmail.com
}

Recibido: 14 de mayo de 2020 / Aceptado: 26 de julio de 2020 https://doi.org/10.17081/just.26.39.4283

\section{Resumen}

Este artículo de reflexión pretende analizar el Sistema General de Pensiones en el Estado Social de Derecho colombiano haciendo énfasis en la situación actual de los trabajadores del sector agrícola, dado que se presumen posibles vulneraciones a sus derechos laborales que consagra la Constitución Política, de forma específica, el derecho a la Seguridad Social en Pensión. Por su parte, aunque la Carta Magna hace referencia en el artículo 48 a la seguridad como derecho irrenunciable, lo enuncia de forma general, pero para el caso de trabajadores agrícolas el contenido jurídico adolece de especificación lo cual puede traer como consecuencia el desconocimiento de sus derechos al no configurarse su labor dentro de las formalidades de un contrato. El enfoque es cualitativo inductivo, el cual permite conocer más a profundidad las particularidades del fenómeno. Así mismo, el método de análisis será hermenéutico pues permite la interpretación de los datos tanto primarios como secundarios con el fin de comprender el contexto actual de los trabajadores agrícolas en relación con el derecho a la pensión.

Palabras ClaVe: Derecho Fundamental, Estado Social de Derecho, Pensión, Seguridad Social, Trabajador Agrícola, Trabajo Decente.

\begin{abstract}
This reflection article aims to analyze the General Pension System in Colombia, emphasizing the current situation of workers in the agricultural sector, given that possible violations of their labor rights are presumed, which are specifically enshrined in the Political Constitution, the right to Social Security in Pension. For its part, although the Magna Carta makes reference in article 48 to security as an inalienable right, it states it in a general way, but in the case of agricultural workers, the legal content lacks specification, which may result in ignorance of its rights as their work is not configured within the formalities of a contract. The approach is qualitative inductive, which allows to know more in depth the particularities of the phenomenon. Likewise, the method of analysis will be hermeneutic as it allows the interpretation of both primary and secondary data in order to understand the current context of agricultural workers in relation to the right to a pension
\end{abstract}

KEYWORDS: Fundamental Law, Social Rule of Law, Pension, Social Security, Agricultural Worker, Decent Work.

Como Citar: Ramirez Acevedo, H. A., \& Pérez Romero, P. S. (2021). Análisis jurídico del régimen de pensiòn en trabajadores agrícolas en relación con el trabajo decente. Justicia, 26(39), 225-236. https://doi.org/10.17081/ just.26.39.4283

+ Artículo de reflexión surgido en el semillero Episteme luris y derivado de proyecto de investigación terminado, perteneciente al grupo de Investigacion Socio - jurídica de la Universidad de Boyacá. 


\section{INTRODUCCIÒN}

El desarrollo del presente trabajo de investigación basado en objetivos1 de protección y garantía de derechos laborales en materia de pensión de trabajadores agrícolas se derivan de forma general en la norma estatal de primer orden y por consiguiente se entienden catalogados dichos derechos a lo largo de su contenido jurídico. Es así que, la Constitución Política, según Gómez S.F. (2007), desde el preámbulo nos enseña que Colombia como "Estado Social de Derecho tiene el objetivo de velar por el cumplimiento, efectividad y la garantía de cada uno de los derechos de los ciudadanos. En este sentido, vemos que los trabajadores tienen derecho a las garantías legislativas en relación a su entorno laboral, tanto en el sector urbano como en el sector rural, en aras de ser objeto del deber constitucional que conlleve a constituir una igualdad jurídica que establezca equilibrio en la sociedad. Así mismo, hablar de un trabajo decente implica tener como base principios fundamentales como lo enuncia Monsalve (2012), "la igualdad, libertad, seguridad y dignidad que se configuran como objetivo principal para lograr rescatar la estabilidad, protección y dignidad humana". (p.8)

Todos los trabajadores cuentan con el derecho fundamental a la Seguridad Social el cual se consagra en la Constitución Nacional en su artículo 48, definiendo que "es un servicio público de carácter obligatorio", en especial mención en relación con el sistema de pensiones que se pretende analizar.

En este contexto, se resalta que el derecho a la Seguridad Social cuenta con criterio de fundamentalidad con el fin de darle una mejor eficacia y eficiencia al sistema pensional. A partir de allí, se generan reformas al contenido legislativo dentro de las cuales están la Ley 100 de 1993 y Ley 797 de 2003. De igual forma nacen líneas jurisprudenciales evidenciadas en fallos de la Corte Constitucional que llevan a considerar "el derecho a la pensión como un derecho prestacional en conexidad con otros derechos fundamentales. Del mismo modo en el contexto de la Seguridad Social se determina como un derecho político, social y económico de mayor trascendencia en el orden constitucional, resaltando que es un servicio público de carácter obligatorio que debe ir acorde con el cumplimiento efectivo de los fines esenciales que debe lograr y ejecutar el Estado Social de Derecho" (Duque Gómez, N y Duque Quintero 2016).

El derecho a la pensión debe ser catalogado como derecho fundamental autónomo teniendo en cuenta que son protegidos por la Constitución Nacional y en esta medida debe ser acatado con el objetivo de generar una protección especial a los afiliados. Sin embargo, es de vital importancia que se vele por la garantía de los derechos de los trabajadores con el objeto de adquirir protección en el momento de presentarse alguna contingencia derivada de la vejez, invalidez y muerte, así como se establece en la (C. Const. SentenCia -T 431/ 2009), "en referencia al derecho a la pensión de invalidez como parte del derecho a la Seguridad Social reitera la jurisprudencia que analiza la Seguridad Social como derecho fundamental". De la misma forma la (C. Const. Sentencia- T 779/2010), lo ratifica como "una garantía que deber ser cumplida sin excepción alguna por tener carácter de fundamental".

Así mismo, en Relación al Régimen de Pensiones en Trabajadores Agrícolas, nuestro ordenamiento normativo cuenta con normas jurídicas que disponen y protegen los derechos fundamentales de este tipo de trabajadores. Aun así, en Colombia no se evidencia una normativa específica para los trabajadores de dicho sector, lo que deja como consecuencia, un vacío normativo sobre este tipo de trabajadores. Por otra parte, sus derechos fundamentales, el reconocimiento de un trabajo digno y la costumbre cultural se han convertido en un formalismo jurídico adoleciendo de un dinamismo práctico.

1 Los objetivos planteados están orientados a: “Analizar la Jurisprudencia de corte constitucional sobre el Régimen de Pensión en trabajadores del sector agrícola" y así mismo, "establecer los lineamientos normativos dados sobre la protección de los derechos laborales en pensión de trabajadores agrícolas". 
En este sentido, con el paso de los años, se ha hecho común la no afiliación o falta de reconocimiento de las prestaciones sociales y en muchos de los casos los trabajadores no se les cubre el "mínimo de derechos y garantías que se encuentran consagrados en la legislación laboral precisamente por la falta de inspección y vigilancia lo que deja como grave consecuencia la carencia de una adecuada protección en la salud, seguridad en el trabajo, cotizaciones a la Seguridad Social que no se realizan por el verdadero monto de lo devengado" (Monsalve, M 2012, p.14). Todo lo anterior, lleva a reflexionar sobre las condiciones de trabajo decente en este tipo de trabajadores.

\title{
II. Resultados
}

\section{Derecho fundamental a la Seguridad Social: referencia especial a la pensión.}

El tema de la Seguridad Social ha sido eje fundamental en la historia del derecho laboral, además de constituirse como base esencial en la sostenibilidad y rescate de los derechos fundamentales de los trabajadores. Así mismo, este campo de la Seguridad Social es muy importante en nuestro contexto colombiano y aún más desde el campo constitucional tal es así, que el derecho a la Seguridad Social, está referido en los artículos 48 y 49 de manera expresa. Por su parte, el artículo 48 hace referencia a dicho derecho en los siguientes términos:

\begin{abstract}
“La Seguridad Social es un servicio público de carácter obligatorio que se prestará bajo la dirección, coordinación y control del Estado, en sujeción a los principios de eficiencia, universalidad y solidaridad, en los términos que establezca la Ley. Se garantiza a todos los habitantes el derecho irrenunciable a la Seguridad Social. El Estado, con la participación de los particulares, ampliará progresivamente la cobertura de la Seguridad Social que comprenderá la prestación de los servicios en la forma que determine la Ley. La Seguridad Social podrá ser prestada por entidades públicas o privadas, de conformidad con la ley. No se podrán destinar ni utilizar los recursos de las instituciones de la Seguridad Social para fines diferentes a ella. La ley definirá los medios para que los recursos destinados a pensiones mantengan su poder adquisitivo constante. (Constitución Política. 1991, p. 19)
\end{abstract}

Colombia como Estado social de derecho, tiene la finalidad de velar y garantizar por todos los derechos fundamentales de cada persona sin exclusión alguna, como se expresa en la Constitución Nacional, en este caso se hace referencia al art 48 que consagrara el derecho a la Seguridad Social en la medida en que este debe garantizarse como un servicio social para la comunidad basado en principios constitucionales que tienen como objeto brindar la efectividad respectiva para el cumplimento de dichas garantías que el Estado brinda a los particulares, así mismo también consagra una de las más importantes garantías como lo es el derecho a la pensión que por consiguiente la normatividad lo declara como aquel que ofrece un soporte a todas las contingencias que son derivadas de la muerte, vejez o invalidez o sobrevivencia. 


\title{
Por su parte, el artículo 49 complementa dicho concepto estableciendo que:
}

\begin{abstract}
"La atención de la salud y el saneamiento ambiental son servicios públicos a cargo del Estado. Se garantiza a todas las personas el acceso a los servicios de promoción, protección y recuperación de la salud. Corresponde al Estado organizar, dirigir y reglamentar la prestación de servicios de salud a los habitantes y de saneamiento ambiental conforme a los principios de eficiencia, universalidad y solidaridad. También, establecer las políticas para la prestación de servicios de salud por entidades privadas, y ejercer su vigilancia y control. Así mismo, establecer las competencias de la Nación, las entidades territoriales y los particulares, y determinar los aportes a su cargo en los términos y condiciones señalados en la ley. Los servicios de salud se organizarán en forma descentralizada, por niveles de atención y con participación de la comunidad. La Ley señalará los términos en los cuales la atención básica para todos los habitantes será gratuita y obligatoria. Toda persona tiene el deber de procurar el cuidado integral de su salud y la de su comunidad. (Constitución Política. 1991, p. 20).
\end{abstract}

Es de alta relevancia mencionar que el derecho fundamental a la Seguridad Social, tiene como objeto generar eficiencia en las garantías constitucionales que determina la ley y junto a ello, la prestación de servicios que hacen parte del poder del Estado pero que es deber del mismo devolverlos como beneficios a cada uno de los administrados.

El derecho a la pensión, además de obtener un componente constitucional basado en principios de universalidad, solidaridad y eficiencia, puede contener un lazo de conexidad con otras garantías previstas en la Norma Suprema, en este caso con el derecho a la salud que también es considerado por la Carta Magna como derecho fundamental universal, pues de lo que se trata es del respaldo de un derecho no contenido como tal en la Constitución, por otro que si lo está de tal modo que se logre las garantías en conjunto que deben ser resguardadas por el Estado en la medida en que son servicios públicos en favor a la comunidad.

De igual forma la jurisprudencia de la (C. Cons. Sentencia C/655-2003), deja entrever en estos mismos términos lo siguiente:

"A este respecto, los artículos 48 y 49 de la Constitución definen la Seguridad Social como un derecho irrenunciable de todos los habitantes y como un servicio público de carácter obligatorio, estas mismas normas precisan que compete al Estado, con la participación activa de los particulares, ampliar progresivamente la cobertura de los servicios según los parámetros que fije el legislador, al tiempo que prohíbe de forma expresa que los recursos de las instituciones de la Seguridad Social se utilicen o se destinen para fines diferentes a ella".

Desde esta lógica, el legislador se pronuncia ante la eficiencia de la Seguridad Social que se consagra en el artículo 48 y se complementó con el artículo 49 anteriormente mencionados, los cuales establecen las garantías de carácter legislativo que figuran en la Constitución Política de Colombia, con el fin de hacer efectivo este derecho fundamental evitando ser trasgredido o afectado por causas como la mala distribución o incorrecta utilización de recursos, que por normatividad deben ser destinados únicamente para fines específicos a garantizar la prestación de los servicios a la población.

En el mismo sentido el Tribunal superior de Cali en su Sala Civil mediante Sentencia 876 de 2017, ha establecido que: 
El derecho a la Seguridad Social cumple con la calidad de ser irrenunciable, el cual se debe a todas las personas sin ninguna exclusión, y en lo que sobreviene la efectividad que gozan los colombianos al acceder al Sistema General de Pensiones, el cual tiene la finalidad de generar una prosperidad a cada uno de los beneficiarios, en la medida en que estos puedan tener una herramienta de ayuda en los casos que tenga que afrontarse la variedad de contingencias derivadas de las enfermedades, la incapacidad laboral, el desempleo, las consecuencias de la muerte, que se imposibilite física o mentalmente para obtener los medios de subsistencia y cualquier otra causa ajena a su voluntad, con el objetivo de brindarle una adecuada protección a ciertos estados propios de la naturaleza humana como la maternidad y la vejez; y a ofrecerle unas condiciones mínimas de existencia y recreación social que le permitan desarrollarse física y sicológicamente en forma libre y adecuada.

La Seguridad Social en primer lugar se constituye como derecho fundamental considerado como servicio público de carácter obligatorio, al cual la población no puede renunciar pues se trata de un derecho que el Estado debe garantizar a todas las personas sin excepción, lo cual conduce a obtener un margen más efectivo en el acceso al Sistema General de Pensiones y que finalmente tendrá como consecuencia el reconocimiento de la pensión sobre los presupuestos establecidos por la ley.

Por otra parte, la Jurisprudencia de la Corte (C. Cons. Sentencia T-317/11), se pronunció en cuanto a la facultad que tiene los beneficiarios para manifestarse cuando se presentan vulneraciones a sus derechos.

“El manejo de los recursos que se obtienen de las cotizaciones o aportes que realizan cada uno de los afiliados, estarán a cargo de las entidades administradoras bien sea de carácter público o privado, por lo tanto en los casos donde se presente una vulneración a dicho derecho fundamental de los afiliados, estos tendrán la facultad de presentar una acción de tutela para que se logre la efectividad, garantía y protección de sus derechos, sin dejar atrás que este derecho fundamental goza de una especial protección constitucional".

En Colombia, los derechos pensionales tienen calidad de ser fundamentales, y según las Corte (C. Cons. Sentencias T-090/09, T-168/09, T-217/09, T-396/09), se estipula que estas garantías ya no necesitan de la teoría de la conexidad para ser catalogados como derechos fundamentales.

Ahora bien, la Corte Constitucional también se pronunció en la (C. Cons. Sentencia T-752 de 2008), reconoce que la pensión es un derecho fundamental, por otro lado, la Sala de Revisión encuentra oportuno señalar, que según la consideración optada en la anterior providencia señalada, la Seguridad Social es un derecho fundamental con componentes de alta relevancia para la comunidad tal como lo establece la Constitución Política en el artículo 48 .2, en consonancia con el artículo $9^{\circ}$ del PIDESC2 (Pacto Internacional de Derechos Económicos, Sociales y Culturales)3.

2 Artículo 9 del PIDESC señala que los Estados Partes en el presente Pacto reconocen el derecho de toda persona a la seguridad social, incluso al seguro social.

3 Ver al respecto, Nogueira Alcalá, Humberto. (2009). Los Derechos Económicos, Sociales y Culturales como derechos fundamentales efectivos en el constitucionalismo democrático latinoamericano. Estudios constitucionales, 7(2), 143-205. https://dx.doi.org/10.4067/S0718-52002009000200007 


\section{Fundamentos jurídicos básicos del régimen de pensión en Colombia}

La fuente esencial es la misma (Constitución Política Art., 48) la cual establece que "La Seguridad Social es un servicio público de carácter obligatorio que se prestará bajo la dirección, coordinación y control del Estado, en sujeción a los principios de eficiencia, universalidad y solidaridad, en los términos que establezca la Ley". (Muñoz O, Esquerra M, 2012. P 89). De tal forma que, la pensión al ser elemento constitutivo de la seguridad social, se cataloga como servicio público obligatorio bajo el auspicio pleno del Estado quien debe garantizar el acceso a dicha prestación sobre la base del contrato realidad con el fin de asegurar el modus vivendi de la sociedad formalizada laboralmente.

Una base importante es también la Ley 100 de 1993, y que al respecto establece lo siguiente:

"El Sistema General de Pensiones tiene por objeto garantizar a la población, el amparo contra las contingencias derivadas de la vejez, la invalidez y la muerte, mediante el reconocimiento de las pensiones y prestaciones que se determinan en la presente ley, así como propender por la ampliación progresiva de cobertura a los segmentos de población no cubiertos con un sistema de pensiones". (Ley 100/ 1993, Art.10).

Así mismo, la obligatoriedad en la afiliación por parte de todos los trabajadores bien sean independientes o dependientes, La selección de los regímenes es voluntaria y libre, lo que antecede que cada uno de los afiliados debe plasmar por escrito dicha elección en el momento de su vinculación o traslado, teniendo en cuenta que si en alguno de los casos donde una persona natural o una persona jurídica desconozca este derecho por alguna situación, se hará conforme a la ley que estipula unas sanciones según lo estipula el artículo 271 de la Ley 100 de 1993.

En síntesis, el Estado Colombiano tiene el deber constitucional de velar y garantizar cada una de las prerrogativas que establece la Carta Magna una de ellas es el derecho fundamental a la Seguridad Social que es un servicio público que debe ser brindado de manera eficaz y eficiente a todos los trabajadores de Colombia sin excepción alguna. De esta forma, las personas que laboran en el sector agrícola gozan plenamente de este derecho de conformidad también con lo expuesto en la Ley 100 de 1993.

En este orden de ideas, se puede establecer que los formalismos normativos están dados, estructurados, son de público conocimiento y se concretan en un escrito pero hasta el momento no se evidencia su funcionalidad práctica, en razón a que la Seguridad Social -aunque es un servicio público y en cierta medida constituye un derecho fundamental en cuanto a que es inherente a la dignidad humana-, pareciera estar supeditada solamente al formalismo del contrato, es decir aquellas acreencias que surgen en razón a la relación laboral y por ende aquellos trabajadores agrícolas al no tener un contrato formalizado se sigue como consecuencia el no reconocimiento de sus prestaciones.

\section{Análisis del contexto pensional en trabajadores agrícolas colombianos}

Adicional a los preceptos constitucionales citados y desde la óptica del Estado Social de Derecho se consagra la obligación de proteger los derechos laborales y el acceso a la Seguridad Social de todos los trabajadores independientemente de su rol y en este sentido también aquellos que hacen parte del sector agrícola y que se prevé en el artículo 64 de la Carta Magna, en el cual se encuentra expuesto de forma general el ámbito de protección a este tipo de trabajadores: 
Es deber del Estado promover el acceso progresivo a la propiedad de la tierra de los trabajadores agrarios, en forma individual o asociativa, y a los servicios de educación, salud, vivienda, Seguridad Social, recreación, crédito, comunicaciones, comercialización de los productos, asistencia técnica y empresarial, con el fin de mejorar el ingreso y calidad de vida de los campesinos. (Constitución Política, 1991. Art 64.)

Tal como lo expresa la Carta Magna el Estado tiene el deber constitucional de garantizar el servicio a la Seguridad Social, con la finalidad de lograr establecer una mejor calidad de vida para el trabajador agrícola, lo que conlleva a que se genere una mayor efectividad en relación al régimen pensional que tiene por objeto, cobijar a la población mediante reconocimiento de una pensión y así garantizar su mínimo vital en la posteridad. Situación que se evidencia también en el espíritu normativo de la Ley 797 de 20034, cuyo objeto radica en conservar y respetar todas las garantías, derechos, servicios y beneficios adquiridos que se aplicaran a todos los habitantes sin excepción alguna.

De igual forma es importante destacar que la Corte Constitucional en la (C. Cons. Sentencia C-1006/ 2005) hace referencia a los beneficios que se otorgan al trabajador del campo, traducidos en un trato particular que ha de ser diferente a los otros sectores de la sociedad, lo que conlleva a establecer un nuevo equilibrio de igualdades jurídicas, económicas, sociales y culturales en especial para la comunidad de trabajadores del agro. En esta línea se manifiesta también la (C. Cons. Sentencia C- 644/2012), la cual hace alusión a las "condiciones de igualdad real para el trabajador del campo", traducidas en la posibilidad de acceder también a los beneficios sociales en virtud de su ejercicio laboral que desarrolla día a día.

Por otra parte, el fomento de dicha actividad agrícola logra establecer la prosperidad de los otros sectores económicos y así mismo, el Estado como garante de los derechos fundamentales debe tener el papel de interventor en este campo de la economía que tiene como finalidad darle un nuevo rumbo de calidad de vida a cada uno de sus ejecutores, debido a que este tipo de trabajadores se encuentra en un estado de miseria y vulneración de sus derechos esenciales, tal contexto se percibe en la (C. Cons. Sentencia C-077/17). En este sentido, el Estado debe actuar como interventor para garantizar el cumplimiento de los derechos constitucionales mediante la realización y ejecución de una protección basada en igualdad jurídica, económica y social con el fin de generar un equilibrio con los otros sectores de la sociedad y mejorar la calidad de vida de los trabajadores agrícolas, teniendo en cuenta que la actividad que cada uno desarrolla en ocasiones es el único sustento para la familia que tienen a su cargo. Por su parte, la (C. Cons. Sentencia T-317/ 2011) establece la importancia de generar la garantía efectiva y protección especial tanto del derecho fundamental a la Seguridad Social como al derecho de orden prestacional el cual, constituye por ejemplo la pensión por vejez y que le garantizan en el futuro su integridad vital5

En esta misma línea se encuentra la (C. Cons. Sentencia T-284/2011), la cual establece que "el derecho a la pensión de vejez reviste un carácter de constitucional como quiera que se deriva directa e inmediatamente de los derechos a la Seguridad Social y al trabajo pues nace y se consolida ligado a una relación laboral. En el mismo sentido, se ha entendido que el derecho a la pensión puede adquirir el carácter de fundamental cuando se encuentra en conexidad con la violación de otro derecho fundamental."

En la Sentencia (C. Cons. Sentencia C-177/1998), definió la Pensión de vejez como "un salario diferido del trabajador, fruto de su ahorro forzoso durante toda una vida de trabajo". Lo que atañe que, "el pago de una

4 Por medio de esta Ley se reforman algunas disposiciones del sistema general de pensiones contenido en la Ley 100 de 1993 y donde se adoptan disposiciones sobre los Regímenes Pensionales exceptuados y especiales.

5 En la Sentencia T-284/07, hace referencia al derecho de pensión de vejez como carácter constitucional y como carácter constitucional, de igual forma resalta que este derecho al reconocimiento y pago para una vida digna se puede consolidar como un derecho fundamental por conexidad. 
pensión no es una dádiva súbita de la Nación, sino el simple reintegro que del ahorro constante durante largos años, es debido al trabajador", (C. Cons. Sentencia C-546 de 1992).

Como vemos el Estado protege y garantiza el derecho al trabajo a cada uno de los colombianos por medio de mandato constitucional consagrado en el artículo 25, que a su tenor manifiesta "El trabajo es un derecho y una obligación social y goza, en todas sus modalidades, de la especial protección del Estado. Toda persona tiene derecho a un trabajo en condiciones dignas y justas"6. Es decir, que cada ciudadano está en todo su derecho de tener un trabajo digno que garantice su mínimo vital y que genere un contexto de igualdad, tal como se define en el artículo 53 de la Constitución"7.

Es claro que desde la perspectiva constitucional los trabajadores, cualquiera que sea su vinculación gozan del derecho de igualdad garantizado por el Estado y que en definitiva es el que debe prevalecer, en la medida en que a todos - en especial mención a los que pertenecen al sector agrícola-, se les debe proporcionar desde el contexto de dignidad y decencia. Es un derecho irrenunciable y aún más tratándose del reconocimiento de la pensión legalmente establecido.

\section{Perspectivas del trabajo decente en los trabajadores del sector agrícola colombiano}

Colombia como Estado Social de Derecho consagra que el derecho al trabajo debe ir conforme a los principios y derechos que se estipulan dentro de la Constitución Política y que garanticen el "modus vivendi" de los trabajadores agrícolas desde el contexto de trabajo decente, aspecto explícito en nuestro ordenamiento jurídico laboral. Por su parte, (Equiza,2003); (Sen y Kliksberg 2007), establecen que:

"cuando las condiciones laborales de los trabajadores se encuentran en estado favorable, se considera que se constituyen en garantía para la Seguridad Social y de igual forma se presentan oportunidades que propicia el bienestar y la dignidad del sujeto con la labor que realiza". (p.30)

En el mismo sentido, hay que resaltar que el ideal de generar una mejor calidad de vida y optimizar las oportunidades en el sector agrícola, es de alta importancia ya que con tal acción, se evidenciaría de forma más práctica un impacto positivo frente a la desigualdad entre los trabajadores del sector rural sin contrato y los trabajadores del sector urbano la mayoría con contrato. Sin embargo, pese a que la normatividad está presente como base reguladora de las garantías y los derechos laborales, es de vital importancia que se evidencie en la praxis.

La decisión del constituyente de 1991 no fue caprichosa en darle al trabajo los calificativos de prevalencia de un interés general, también le dio la importancia que merecía dentro de la estructura democrática, de igual forma lo calificó como principio, derecho fundamental y obligación social que goza en todas sus modalidades de especial protección estatal, teniendo en cuenta que el derecho al trabajo es un valor fundante del Estado Social de Derecho, que hace posible la dignificación del ser humano y desde luego se convierte en un elemento básico para el cumplimiento de los fines esenciales del Estado.

Desde la perspectiva del trabajo decente en el sector agrícola se puede inferir que existe, por un lado, deficiencia de servicios sociales y por otro lado, simplemente no se presta el servicio adecuado, más aun, no figuran incluidos en el Sistema de Seguridad Social por parte del Estado Colombiano.

6 En el artículo citado, es importante el derecho fundamental al trabajo y de igual forma establece una obligación social que debe respetarse y acatarse por todos los colombianos sin excepción alguna.

7 En el artículo 53 anteriormente citado, establece que el Congreso expedirá el estatuto del trabajo, garantizando las respectivas prerrogativas al derecho fundamental al trabajo para toda la sociedad. 
De igual forma, el sector agrícola es una de las poblaciones que más están expuestas a riesgos teniendo en cuenta que se ven afectados en el plano social. En este sentido, Ron Oswaldo, (Secretario General de la Unión Internacional de Trabajadores de la Alimentación, Agrícolas, Hoteles, Restaurantes, Tabaco y Afines) (UITA), ha mencionado que "Estamos firmemente convencidos que la ausencia de derechos en el sector agrícola es la principal causa del déficit de trabajo decente". (Baker, 2003, p. 6). No solo se plasma notoriamente la falta de asistencia por parte del Estado y garantía de un trabajo decente para los trabajadores agrícolas, si no también se evidencia la carencia de políticas estatales que respondan a las necesidades básicas sociales de dicho sector.

Desde esta óptica, se puede concluir que desde el contexto social el sector agrícola se encuentra en una gran desventaja con respecto a los demás sectores de la economía colombiana y dado que como lo afirma Alcocer ( 2013 )“El hombre que trabaja en el campo es el motor del progreso de la sociedad en cuanto la provee de alimentos para la sobrevivencia de la especie y la materia prima para las labores industriales; por tanto, es labor prioritaria de esta misma sociedad garantizarle su seguridad integral" ( p. 2).

Así mismo, el trabajo en el sector agrícola en Colombia y en la mayoría de los países de América Latina y el Caribe se caracterizan por estar en malas condiciones laborales y vulneración de los derechos fundamentales de los trabajadores, en cuanto que se dificulta el acceso a los servicios básicos que el Estado otorga como lo es el acceso a la Seguridad Social, lo cual conduce a que el trabajador agrícola no tenga la posibilidad de mejorar su contexto vital, sus condiciones laborales y sus garantías jurídicas según lo estipulado constitucionalmente. Por lo tanto, es importante resaltar la obligatoriedad que debe ejercer el Estado en el momento de la prestación de los servicios.

De conformidad con un estudio de la Oficina Internacional del Trabajo (en adelante O.I.T) acerca del trabajo decente y productivo en la agricultura estableció que:

\begin{abstract}
La agricultura es importante como fuente de empleo e ingresos, en los países de desarrollo. Sin embargo, los trabajadores agrícolas suelen enfrentar déficit de trabajo decente, trabajar por un salario y unos ingresos escasos en condiciones a veces peligrosas, y carecer de medios para abordar su situación de forma eficaz. (OIT, 2017, p2)
\end{abstract}

En este sentido, el trabajo decente también debe ser garantizado teniendo en cuenta la forma de contratación, la forma de pago y la protección social que el empleador le ofrece al trabajador, los cuales son esenciales para la construcción de una base sólida que garantice el bienestar y el trabajo digno de cualquier trabajador es por ello que "la relación estrecha entre el trabajador y el empleador constituyen un elemento esencial para el desarrollo humano, que posibilita a las personas llevar el tipo de vida que desean, sueñan y valoran" (Puello, Ramos, Madariaga,(2012, p.23). Es importante destacar, que si este vínculo se da conforme a la garantía de derechos adquiridos de los cuales gozan los trabajadores agrícolas, se podrá lograr mejorar la productividad laboral y la calidad de vida de cada uno de ellos. Hay que mencionar además que la Corte Constitucional estableció que "En el marco de la OIT se distinguen dos aspectos: por un lado, el derecho que rige la relación entre empleador y trabajador, y por otro, las previsiones legislativas orientadas a garantizar las condiciones del trabajo decente". (C. Const. Sentencia C- 645/11).

Así mismo, la OIT establece los elementos esenciales del trabajo decente que tienen como objetivo "promover oportunidades para que los hombres y las mujeres puedan obtener un trabajo decente y productivo en condiciones de libertad, equidad, seguridad y dignidad humana". (Somavia, 1999), efectivamente con el cumplimiento de cada uno de los principios que plantea la OIT se puede deducir que los trabajadores que se encuentren ejerciendo su labor en beneficio de la comunidad, ya sea de forma remunerada por me- 
dio de un salario o a destajo o por jornal etc., se les debe garantizar siempre sus derechos conforme a las formalidades que exige "un trabajo decente".

Por consiguiente, en Colombia hoy en día se sigue evidenciado un déficit de trabajo decente en los trabajadores del sector agrícola, por falta de formalización del empleo en dicho sector y que este conforme a lo que dispone la Ley. Desde este panorama no es posible pensar en la consistencia de un trabajo decente debido a que hoy en día, no obstante, de que existe la normatividad y los formalismos respectivos que establecen acceso y garantía a un Sistema de Seguridad Social, no se constata en la realidad dicha garantía como tampoco el respeto de los principios mínimos fundamentales que estipula un verdadero trabajo decente en los trabajadores y en especial mención a los del sector agrícola.

El tipo de contratación, la forma de pago y la protección social etc, que se ofrecen los empleadores, deben ser elementos esenciales que ayuden al aumento de la calidad de vida, construcción de armonía personal, familiar y social de cada uno de los trabajadores del sector agrícola. En este sentido, se nota una estrecha relación entre la realización del trabajador y la calidad laboral, lo cual facilita que este tipo de población lleve un estilo de vida más adecuada y ajustada a los principios de nuestra constitución. Equizá (2003) "afirma que en el momento en que los trabajadores realizan su labor en condiciones buenas y conforme a sus garantías paralelamente se está propiciando el bienestar de estos colectivos humanos".

\section{Conclusiones}

Según la Ley 100 de 1993, en su artículo 10 hace referencia al objeto del Sistema General de Pensiones el cual consiste en garantizar a los trabajadores el amparo contra las contingencias derivadas de la vejez, la invalidez y la muerte, mediante el reconocimiento de las pensiones y prestaciones que se determinan en dicha Ley. Dado que, en principio la formalidad normativa es explicita y además es deber constitucional velar por su garantía y cumplimiento, en la práctica se evidencia lo contrario a lo estipulado.

El desconocimiento del régimen de Seguridad Social facilita la vulneración de derechos laborales sobre todo en la población laboral agrícola, de igual forma el acceso a la jurisdicción laboral y el trámite procesal se constituyen en acciones engorrosas para el trabajador, el cual prefiere percibir solamente un salario diario o semanal o quincenal y dejar al margen las prestaciones sociales, salud, pensión, riesgos laborales, servicios complementarios etc.

En relación al primer objetivo planteado, la Corte Constitucional, ha venido analizando cómo el sistema jurídico colombiano ha construido el corpus iuris respecto a los trabajadores campesinos y agrícolas, el cual reconoce derechos, deberes y principios constitucionales que son acordes a la garantía de la dignidad humana, la cual es característica esencial y se constituye en principio dentro de nuestro ordenamiento tal como lo menciona la (C. Const. Sentencia C- 028/2018), “La dignidad humana entendida como autonomía de diseñar un plan vital y de determinarse según sus características (vivir como quiera), La dignidad humana entendida como ciertas condiciones materiales concretas de existencia (vivir bien), y la dignidad humana entendida como intangibilidad de los bienes no patrimoniales, integridad física e integridad moral (vivir sin humillaciones)". Sin embargo, se puede inferir que la mayoría de trabajadores del sector agrícola, no tienen conocimiento respecto al conceptos como "trabajo decente" en el contexto jurídico- laboral, derechos fundamentales, dignidad humana, antes bien, los conceptos los asocian con significados puramente éticos y morales. 
Respecto al segundo objetivo planteado, los lineamientos normativos establecidos sobre protección de derechos laborales en pensión de trabajadores agrícolas, están establecidos de forma general en la Constitución Política, desde el punto de vista de principios, valores y derechos esenciales. De igual forma en la Ley ordinaria, la cual hace referencia en su contenido normativo a todo el conjunto de trabajadores, sin hacer especificaciones. Por su parte la jurisprudencia de las altas Cortes, coinciden en asegurar la observancia y la garantía de los derechos laborales de forma más explícita a los trabajadores del campo, pero en relación con sus prerrogativas constitucionales. Es decir, que para este tipo de trabajadores no existe un régimen especial, anexo, diferente al que se ha establecido para los trabajadores formalizados. Así, las prestaciones sociales, en este caso la pensión, será solamente un ideal, pues los trabajadores del campo al adolecer de claridad normativa y especial protección no van a cotizar y estarán supeditados a las condiciones del empleador, lo cual trae como consecuencia la vulneración de sus derechos y contradicción respecto a las exigencias de la Organización Internacional del Trabajo (OIT) en relación a "trabajo decente".

\section{Referencias Bibliográficas}

Baker, J; (2003). El Trabajo Decente en la Agricultura, Documento de Referencia, Simposio Internacional de Trabajadores Sobre el Trabajo Decente en la Agricultura, Oficina de Actividades para los Trabajadores de la OIT, Ginebra. Descargado 23 de junio de 2020. En: www.fediap.com.ar > administracion > pdfs > ElTrabaj...

Corte Constitucional Colombiana. Sentencia C-546 de 1992. M.P. Ciro Angarita Barón.

Corte Constitucional Colombiana. Sentencia C-177 de 1993. M.P. Alejandro Martínez Caballero.

Corte Constitucional Colombiana. Sentencia C-655 de 2003. M.P. Rodrigo Escobar Gil.

Corte Constitucional Colombiana. Sentencia C-1006 de 2005. M.P. Álvaro Tafur Galvis.

Corte Constitucional Colombiana. Sentencia T-752 de 2008. M.P. Humberto Antonio Sierra Porto.

Corte Constitucional Colombiana. Sentencia T-431 de 2009. M.P. Humberto Antonio Sierra Porto.

Corte Constitucional Colombiana. Sentencia T-779 de 2010. M.P. Humberto Antonio Sierra Porto.

Corte Constitucional Colombiana. Sentencia T-317 de 2011. M.P. Jorge Iván Palacio Palacio.

Corte Constitucional Colombiana. Sentencia T-284 de 2011. M.P. Manuel José Cepeda Espinoza.

Corte Constitucional Colombiana. Sentencia C-645 de 2011. M.P. Gabriel Eduardo Mendoza.

Corte Constitucional Colombiana. Sentencia C- 644 de 2012. M.P. Adriana María Guillen Arango.

Corte Constitucional Colombiana. Sentencia C- 077 de 2017. M.P. Luis Ernesto Vargas Silva.

Tribunal superior Sala Civil de Cali, Sala de Casación Civil y Agraria. STC/8762-2017. MP. Álvaro Fernando García Restrepo.

Equiza, F. (2003). Amartya Sen y sus aportaciones a la Teoría del Desarrollo: Algunas reflexiones. Descargado 23 de junio de 2020. En: http:// www.gobernabilidad.cl/modules. php?name=News\&file=print\&sid=139[1Septiembre 2010]. En: Puello Alcocer, E., Ramos, J., \& Madariaga, C. (2012). Condiciones laborales de los trabajadores agrícolas del municipio de Montería, Colombia. Temas Agrarios, 17(1), 20-31. https://doi.org/10.21897/rta.v17i1.693.

Gómez, S. F. (2007). Constitución Política de Colombia. Bogotá, Colombia. LEYER.

Ley 100 de 1993. Por la cual se crea el sistema de seguridad social integral y se dictan otras disposiciones. Diciembre 23 de 1993.D.O. N 41.148. 
Ley 797 de 2003. Por la cual se reforman algunas disposiciones del sistema general de pensiones previsto en la Ley 100 de 1993 y se adoptan disposiciones sobre los Regímenes Pensionales exceptuados y especiales. Enero 29 de 2003. DO. N 45.079

Organización Internacional de Trabajo; (2017). Trabajo Decente y Productivo en la Agricultura. Descargado el 20 de junio de 2020. En: https://www.ilo.org/wcmsp5/groups/public/ed_dialogue/--sector/ documents/...

Puello Alcocer, E., Ramos, J., \& Madariaga, C. (2012). Condiciones laborales de los trabajadores agrícolas del municipio de Montería, Colombia. Temas Agrarios, 17(1), 20-31. Descargado el 19 de junio de 2020. En: https://doi.org/10.21897/rta.v17i1.693

Sen, A. y Kliksberg, B. (2007). Primero la gente. Una Mirada desde la ética del desarrollo a los principales problemas del mundo globalizado. Ediciones Deusto EE, España, p.30. En: Puello Alcocer, E., Ramos, J., \& Madariaga, C. (2012). Condiciones laborales de los trabajadores agrícolas del municipio de Montería, Colombia. Temas Agrarios, 17(1), 20-31. https://doi.org/10.21897/rta.v17i1.693

Somavia, J; (1999). Organización Internacional del Trabajo. Descargado el 26 de julio de 2018, En: http://www. ilo.org/public/spanish/bureau/dgo/speeches/somavia/1999/seattle.htm\#note1. 